\title{
Tracing the power-law component in the energy spectrum of black hole candidates as a function of the QPO frequency
}

\author{
F. Vignarca ${ }^{1,2}$, S. Migliari ${ }^{1,4}$, T. Belloni ${ }^{1}$, D. Psaltis ${ }^{3}$, and M. van der Klis ${ }^{4}$ \\ 1 INAF - Osservatorio Astronomico di Brera, Via E. Bianchi 46, 23807 Merate (LC), Italy \\ 2 Dipartimento di Scienze Università dell'Insubria, via Valleggio, 22100 Como, Italy \\ 3 School of Natural Sciences, Institute for Advanced Study, Princeton, NJ 08540, USA \\ 4 Astronomical Institute "Anton Pannekoek" University of Amsterdam and Center for High-Energy Astrophysics, \\ Kruislaan 403, NL 1098 SJ Amsterdam, Netherlands
}

Received 12 July 2002 / Accepted 18 October 2002

\begin{abstract}
We investigated the relation between the centroid frequency of the quasi-periodic oscillation observed in the power density spectra of a sample of galactic black-hole candidates with the power-law photon index obtained from spectral fits. Our aim is to avoid inner accretion disk radius determination directly from spectral fits, given the uncertainties of the absolute values obtained in that way, but to base our analysis on the likely association of QPO frequency to a characteristic radius. We used archival RXTE data of GRS 1915+105 and published parameters for GRO 1655-40, XTE J1550-564, XTE J1748-288 and 4U 1630-47. While for low values of the QPO frequency, the two parameters are clearly correlated for each source, there is evidence for a turnoff in the correlation above a characteristic frequency, different for different sources. We discuss the possible nature of this turnoff.
\end{abstract}

Key words. accretion: accretion disks - black hole physics - stars: oscillations - X-rays: stars

\section{Introduction}

In 1992, the WATCH all-sky monitor onboard GRANAT discovered the X-ray source GRS $1915+105$ as a bright transient (Castro-Tirado et al. 1992). Mirabel \& Rodríguez (1994) reported the observation of radio relativistic expansions interpreted in terms of collimated emissions of matter, which made GRS 1915+105 the first galactic object with superluminal jets, for which the term "microquasar" was coined. This source was originally supposed to host a black hole because of its high $\mathrm{X}$-ray luminosity, and for its similarity with another superluminal source, GRO J1655-40, for which it was possible to obtain a dynamical estimate of the mass $\left(M=6.3 \pm 0.5 M_{\odot}\right)$ of the compact object (Bailyn et al. 1995; Greene et al. 2001). No optical counterpart of GRS 1915+105 was found because of the high Galactic extinction and only an IR counterpart was detected (Mirabel et al. 1994). Recently, an orbital period of 33.5 days was discovered with IR spectroscopic observations, leading to a dynamical estimate of $14 \pm 4 M_{\odot}$ for the compact object (Greiner et al. 2001, see also Borozdin et al. 1999). The numerous observations of GRS $1915+105$ made with the instruments onboard the Rossi X-ray Timing Explorer (RXTE) satellite show an extremely complex variability in the X-ray band, which Belloni et al. (1997a, 1997b) interpreted as

Send offprint requests to: T. Belloni,

e-mail: belloni@merate.mi .astro.it an oscillation of the inner region of the accretion disk caused by a thermal-viscous instability. Belloni et al. (2000) introduced a model-independent classification of the different types of X-ray variability in three basic spectral states. Similarly to more conventional Black Hole Candidates (BHC), the energy spectrum of GRS $1915+105$ can be fitted with the superposition of a soft thermal component, modeled as a disk-blackbody (Mitsuda et al. 1984), plus a harder component, modeled as a power law with a high energy cutoff (Tanaka \& Lewin 1995). The state transition can be attributed to changes in the relative contributions of these variable components, again similarly to what observed in other BHCs (Belloni 1998).

Detailed timing analysis of the RXTE data (Morgan et al. 1997) revealed three types of Quasi Periodic Oscillations (QPO): a 1-10 Hz QPO with variable centroid frequency similar to that observed in other BHCs (see van der Klis 1995), two quasi-stable QPOs with centroid frequencies $\sim 40$ and $\sim 67 \mathrm{~Hz}$ (Morgan et al. 1997; Belloni et al. 2001; Strohmayer et al. 2001a), and variable low frequency QPOs $\left(10^{-3}-1 \mathrm{~Hz}\right)$, which can be identified with transitions between the states of Belloni et al. (2000). The presence of the $1-10 \mathrm{~Hz}$ is always associated with a spectral state $\mathrm{C}$ (Belloni et al. 2000). The $1-10 \mathrm{~Hz}$ QPO centroid frequency is well correlated with both disk and power-law spectral components (Muno et al. 1999; Reig et al. 2000; Rodriguez et al. 2002) and with the X-ray flux (Chen et al. 1997; Markwardt et al. 1999). 
Table 1. List of plateau observations analyzed and results for timing and spectral parameters. Letters a, b, c correspond to different orbits in the same observation. The fifth column refers to the state-C length (in seconds) that we selected for our study. QPO frequencies are in Hertz, $N_{\mathrm{H}} \times 10^{22} \mathrm{~cm}^{-2}$. Typical errors are: for $N_{\mathrm{H}} \pm 0.1$ and for $E_{\mathrm{c}} \pm 0.45$.

\begin{tabular}{|c|c|c|c|c|c|c|c|c|c|c|}
\hline Obs. N & Observation ID & MJD & $\mathrm{PCA}(\mathrm{c} / \mathrm{s})$ & $\Delta T$ & $v_{\mathrm{QPO}}$ & Tim. Res. & $\Gamma$ & $N_{\mathrm{H}}$ & $E_{\mathrm{c}}$ & $\chi_{\mathrm{r}}^{2}$ \\
\hline 1 & $10408-01-23-00 a$ & 50278.4921 & 9735 & 3072 & $3.491 \pm 0.005$ & $1 / 512$ & $2.62 \pm 0.01$ & 6.7 & 24.7 & 1.7 \\
\hline 2 & $10408-01-23-00 b$ & 50278.5585 & 9710 & 3540 & $3.617 \pm 0.004$ & $1 / 512$ & $2.66 \pm 0.01$ & 6.9 & 25.4 & 1.7 \\
\hline 3 & $10408-01-23-00 c$ & 50278.6266 & 10458 & 3401 & $4.199 \pm 0.009$ & $1 / 512$ & $2.76 \pm 0.01$ & 7.1 & 28.0 & 1.7 \\
\hline 4 & $10408-01-24-00 a$ & 50280.1702 & 8924 & 2497 & $2.228 \pm 0.008$ & $1 / 512$ & $2.38 \pm 0.04$ & 6.4 & 25.1 & 1.7 \\
\hline 5 & $10408-01-24-00 b$ & 50280.2266 & 8901 & 3479 & $2.293 \pm 0.007$ & $1 / 512$ & $2.41 \pm 0.04$ & 6.3 & 23.0 & 1.6 \\
\hline 6 & $10408-01-24-00 c$ & 50280.2933 & 8956 & 2998 & $2.534 \pm 0.008$ & $1 / 512$ & $2.46 \pm 0.04$ & 6.6 & 23.0 & 1.4 \\
\hline 7 & $10408-01-27-00 a$ & 50290.5774 & 7887 & 2421 & $0.642 \pm 0.004$ & $1 / 512$ & $1.88 \pm 0.04$ & 6.9 & 16.2 & 1.3 \\
\hline 8 & $10408-01-27-00 b$ & 50290.6322 & 7926 & 3480 & $0.628 \pm 0.004$ & $1 / 512$ & $1.82 \pm 0.01$ & 6.7 & 15.1 & 1.2 \\
\hline 9 & $20402-01-49-01$ & 50730.3949 & 8198 & 3463 & $2.637 \pm 0.005$ & $1 / 512$ & $2.45 \pm 0.01$ & 5.4 & 29.7 & 1.8 \\
\hline 10 & $20402-01-50-00$ & 50735.5474 & 6579 & 1962 & $0.835 \pm 0.010$ & $1 / 128$ & $1.92 \pm 0.02$ & 5.7 & 21.5 & 1.9 \\
\hline 11 & $20402-01-50-01 a$ & 50737.4047 & 6608 & 3007 & $1.014 \pm 0.006$ & $1 / 128$ & $1.98 \pm 0.01$ & 5.7 & 22.1 & 1.6 \\
\hline 12 & $20402-01-50-01 b$ & 50737.4766 & 6373 & 2620 & $1.078 \pm 0.005$ & $1 / 128$ & $2.00 \pm 0.01$ & 5.5 & 20.0 & 1.6 \\
\hline 13 & $20402-01-52-00 a$ & 50746.5509 & 6657 & 2207 & $1.405 \pm 0.007$ & $1 / 128$ & $2.09 \pm 0.04$ & 5.5 & 25.0 & 1.8 \\
\hline 14 & $20402-01-52-00 b$ & 50746.6190 & 6668 & 2079 & $1.468 \pm 0.006$ & $1 / 128$ & $2.10 \pm 0.04$ & 5.5 & 25.1 & 1.4 \\
\hline 15 & $20402-01-52-00 c$ & 50746.6898 & 6768 & 1725 & $1.609 \pm 0.007$ & $1 / 128$ & $2.16 \pm 0.04$ & 5.6 & 26.9 & 1.7 \\
\hline
\end{tabular}

Several theoretical models associate the origin of this QPO with the innermost region of the disk (Miller et al. 1998; Nowak \& Wagoner 1991; Titarchuk et al. 1998; Stella \& Vietri 1998; Stella et al. 1999; Varnière et al. 2002; Psaltis \& Norman 2002). Di Matteo \& Psaltis (1999) made use of the fact that the fastest variability timescale at any radius around a compact object is the Keplerian orbital frequency to obtain upper limits on the radius at which the $1-10 \mathrm{~Hz}$ QPO is produced. As it is apparent in their work, there is a general positive correlation, considering different systems, between the photon index of the power-law component in the energy spectrum and the centroid frequency of the QPO. Sobczak et al. (2000a) in their analysis of RXTE data of two transient BHCs, XTE J1550-564 and GRO J1655-40, examined the correlation between the spectral parameters, inner disk radius and power-law photon index, with the QPO centroid frequency. Surprisingly, they find correlations of opposite sign for the two sources. Rodriguez et al. (2002) analyzed the correlation between QPO centroid frequency and inner disk radius (determined from X-ray spectral fits).

In this paper, we concentrate on the correlation between power-law photon index and QPO centroid frequency. We present the results of timing and spectral analysis of a number of RXTE observations of GRS 1915+105, focusing on this correlation. We also consider published data relative to four other systems: GRO J1655-40 (Remillard et al. 1999a; Sobczak et al. 2000a), XTE J1550-564 (Sobczak et al. 2000a,b; Homan et al. 2001), XTE J1748-288 (Revnivtsev et al. 2000), 4U 1630-47 (Trudolyubov at al. 2001; Tomsick \& Kaaret 2000). We then compare the results in term of possible theoretical models.

\section{Data analysis}

Our study of GRS $1915+105$ is divided in two parts, according to the different classes of observations that we considered. In the first part, we analyze the so-called plateau observations
(Fender et al. 1999; Reig et al. 2000), which consist in long intervals of persistent state C (class $\chi$ in Belloni et al. 2000). The second part is dedicated to observations that show large variability in the X-ray flux and at least one state-C interval. Again following the classification of Belloni et al. (2000), we selected observations corresponding to classes $\alpha, \beta$, and $\nu$.

\subsection{Plateau observations}

Plateau intervals show persistent QPOs that are very easily detectable in the Power Density Spectrum (PDS). We based our choice of appropriate observations upon the extensive analysis of Reig et al. (2000), which allowed us to identify the larger possible range of centroid frequencies of the QPO. We selected 7 RXTE/PCA observations of GRS 1915+105 (for a total of 15 satellite orbits), corresponding to class $\chi$ in the classification of Belloni et al. (2000). The observation log is reported in Table 1.

The plateau observations are relatively straightforward to analyze from the point of view of timing analysis, because of the stable positive correlation between the QPO frequency and count rate (see Reig et al. 2000). Therefore, no special timing selection is necessary and it is possible to accumulate all the data to produce one PDS and one energy spectrum. To produce the PDS, for each observation we used high-time resolution data in the $2-13 \mathrm{keV}$ range. Some of the observations have a time resolution of $1 / 512 \mathrm{~s}$, limited to $1 / 128 \mathrm{~s}$ for the others (see Table 1). We divided each observation interval (orbit of the satellite) in intervals $16 \mathrm{~s}$ long and produced an average PDS per observation summing the PDS of the individual segments. We subtracted the contribution due to Poissonian statistics and the Very Large Event window (Zhang et al. 1995; Zhang 1995) from each PDS and renormalized them to squared fractional rms per Hertz (see Belloni \& Hasinger 1990). Every PDS was then logarithmically rebinned and fitted to find the 

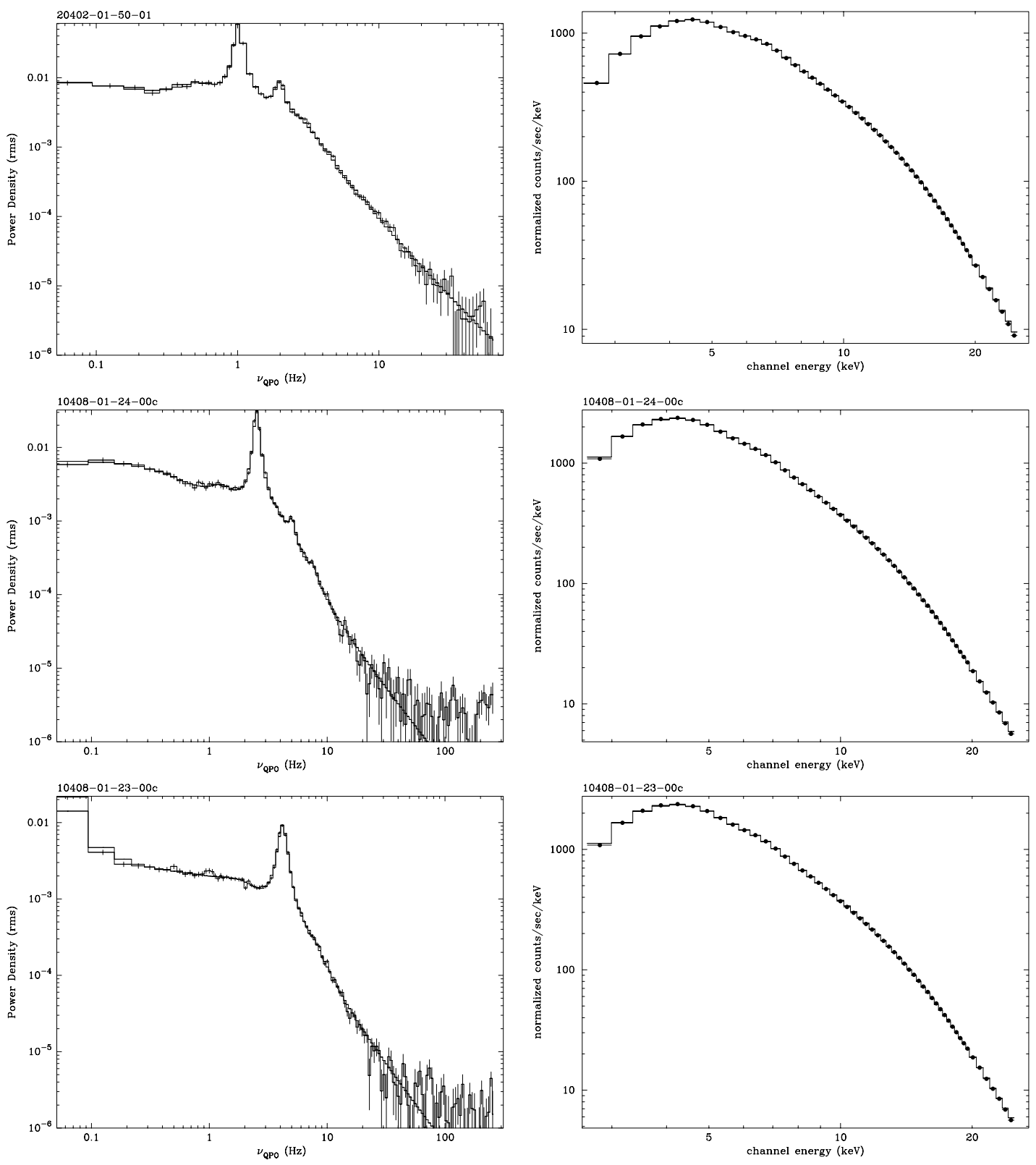

Fig. 1. Examples of power density spectra (left panels) and energy spectra (right panels) for three plateau observations with different QPO frequency values.

centroid frequency of the $1-10 \mathrm{~Hz} \mathrm{QPO}$, which is very sharp and often shows higher harmonics and even a sub-harmonic component (as shown in Fig. 1 for three different observations). A model consisting of the superposition of a number of Lorentzians (from 5 to 9 depending on the observation) was used to fit every feature present in the power spectrum (see Belloni et al. 2002). Because of the relative strength of the QPO peak, the centroid frequency did not change appreciably when using different models for the continuum noise: the QPO frequencies we found with this analysis are in close agreement with those from Reig et al. (2000). The resulting QPO centroid frequencies are listed in Table 1.

In Class $\chi$ observations, the source is in a long steady state C (Belloni et al. 2000). As shown by Belloni et al. (2000), this corresponds to an undetectable thermal component in the
PCA energy spectrum. For each observation interval we extracted PCA energy spectra in the range 3-25 keV, averaged over the whole interval, from spectral (Standard2) data. For each spectrum, we subtracted the background estimated with pcabackest v. 2.1e, and created an appropriate detector response matrix with pcarsp v. 2.43. A systematic error of $1 \%$ was added to the spectra to account for remaining uncertainties in the detector calibration. Our starting model for the spectral fits consisted of a power law, corrected for interstellar absorption, plus a Gaussian emission line to take into account an excess at $6.4 \mathrm{keV}$. With this model, it was not possible to lower the reduced $\chi^{2}$ below 2 . We modified the model, adding a high-energy cutoff to the power law. The resulting spectral parameters are shown in Table 1. This model is consistent with that used by Trudolyubov et al. (1999) and Muno et al. (1999). 


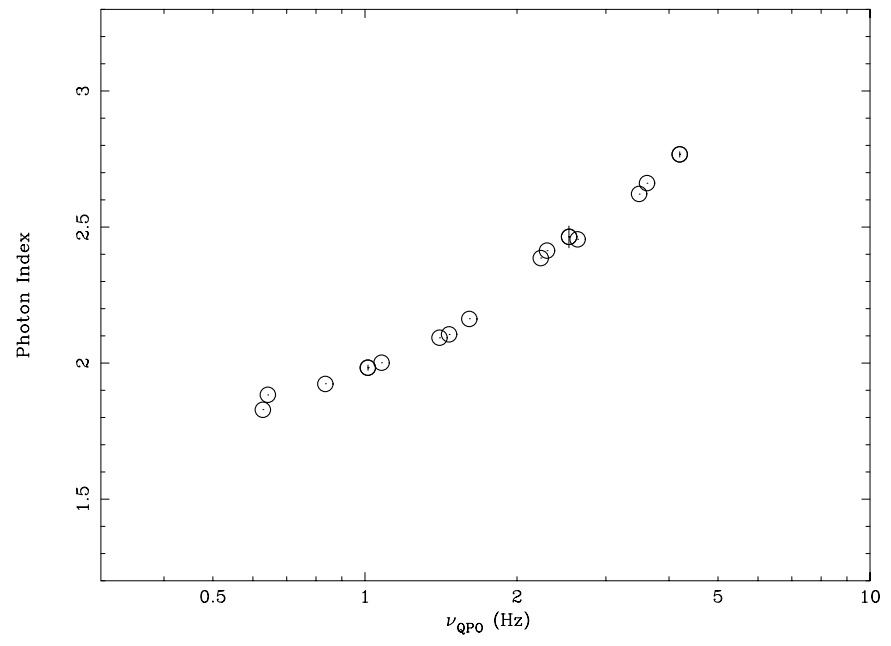

Fig. 2. Plot of power law photon index versus QPO centroid frequency for the plateau observations of GRS 1915+105 studied. A positive correlation spanning nearly an order of magnitude is evident. Representative error bars are plotted for three points.

Notice that Trudolyubov et al. (1999) found rather high cutoff energies ( $100 \mathrm{keV})$, while Muno et al. (1999) found also much lower values, even below $10 \mathrm{keV}$. Our values are in the range 20-30 keV. Only 2 among the selected plateau observations (10408-01-27-00 and 20402-01-52-00) were also analyzed by Muno et al. (1999). They used an often-used model for Black Hole Candidates, which consists of a superposition of a multicolor disk-blackbody (thermal component) and a power law (without high energy cutoff). Their spectral analysis results in a different power law photon index, $\sim 0.7$ grater than the values we found. However, they derive an extremely high inner disk temperature $(\sim 4 \mathrm{keV})$ and an inner disk radius consistent with zero $(\sim 2 \pm 2 \mathrm{~km})$. These parameters do not represent a realistic physical picture of the source and therefore suggest that the inclusion of a disk-blackbody component is not physically necessary. Our choice of a simple power law with a high-energy cutoff is motivated by two reasons: the need of a simple model for efficiently performing a large number of fits, and the need to use a common model that allows comparison with values in the literature. In order to check that for high values of the spectral index $\Gamma$ this model yields meaningful values, we fitted our spectra also with the bmc model in XSPEC, which has been used by other authors to describe the energy spectra of Black Hole Candidates in the framework of Comptonization models. These fits give values of $\Gamma$ consistent with those from a simple power-law model, with a slight systematic excess of 3\%. From Table 1, one can see that there are variations in the best fit values of the column density $\left(N_{\mathrm{H}}\right)$ (see also Belloni et al. 2000; Klein-Wolt et al. 2002).

In Fig. 2 we plot the power-law photon index versus the QPO centroid frequency. A positive correlation is evident.

\subsection{Class $\alpha, \beta$ and $v$ observations}

In order to examine the correlation between QPO centroid frequency and power-law photon index, and in particular to extend it to higher values of the QPO frequency, we also analyzed
Table 2. List of the observations from classes $\alpha, \beta$ and $v$ analyzed in this work. Letters a, b, c correspond to different observation intervals within the same observation. The last column refers to the length $(\Delta T)$ of the state-C interval that we selected for our study.

\begin{tabular}{clccc}
\hline \hline Obs. N & Obs. ID & MJD & Class & $\Delta T$ \\
\hline 15 & $10408-01-44-00 a$ & 50381.4942 & $v$ & 1248 \\
16 & $10408-01-44-00 b$ & 50381.5655 & $v$ & 1488 \\
17 & $20186-03-03-01 b$ & 50674.2347 & $\beta$ & 704 \\
18 & $20187-02-01-00 b$ & 50575.9322 & $\alpha$ & 928 \\
19 & $40702-01-02-00 b$ & 51320.9322 & $v$ & 1120 \\
\hline
\end{tabular}

observations in which GRS 1915+105 shows large variability on time scales longer than $1 \mathrm{~s}$. While belonging to different classes, they all have in common the presence of at least one state-C interval of more than $700 \mathrm{~s}$ (see Belloni et al. 2000). The log of selected observations is reported in Table 2. After a close inspection of the light curves produced from Standard1 data (corresponding to the full PCA energy band 2-60 keV), we identified the start and end times of the state-C intervals to analyze. Flux values during these periods typically range from 3000 to $40000 \mathrm{cts} / \mathrm{s}$. For each observation, we checked the choice of intervals by producing a dynamical PDS (a series of PDS as a function of time), with which we verified that a 1-10 Hz QPO was present throughout the whole interval. The dynamical PDS also confirmed that the X-ray intensity of the source is strongly correlated with the QPO frequency (Chen et al. 1997; Markwardt et al. 1999; Reig et al. 2000). Therefore, following the PCA count rate, the QPO frequency first decreases rather fast, then starts increasing at a lower rate (see Fig. 4).

To perform timing analysis, we inspected the same type of data (high-time resolution and low energy range) as for plateau observations. In this case however, we divided every state-C interval in segments with a length of $4 \mathrm{~s}$ rather than 16 , in order to better follow the rapid variations of the QPO centroid frequency, and produced a PDS for every segment. After subtracting the contribution from the Poissonian statistics and the Very Large Event window, we summed these PDS in sets of four, rebinned them logarithmically and fitted the result to find the QPO centroid frequency. As in the previous case, a model consisting of the superposition of a number of Lorentzians was used (see Belloni et al. 2002). We performed the summing process because we wanted to correlate timing and spectral results (which have a minimum time bin of $16 \mathrm{~s}$ ), and this is possible only if we analyze intervals with temporal coincidence.

Spectral parameters were obtained extracting energy spectra from Standard2 data, with an integration time of $16 \mathrm{~s}$, subtracting the background and creating the detector response matrix as for the plateau observations. We fitted every spectrum using the "standard" model for BHCs: the superposition of a multicolor disk-blackbody and a power law for the high energy tail. We corrected for the interstellar absorption, fixing $N_{\mathrm{H}}=7 \times 10^{22} \mathrm{~cm}^{-2}$ (Klein-Wolt et al. 2002) and added a Gaussian emission line with central energy fixed to $6.4 \mathrm{keV}$. 

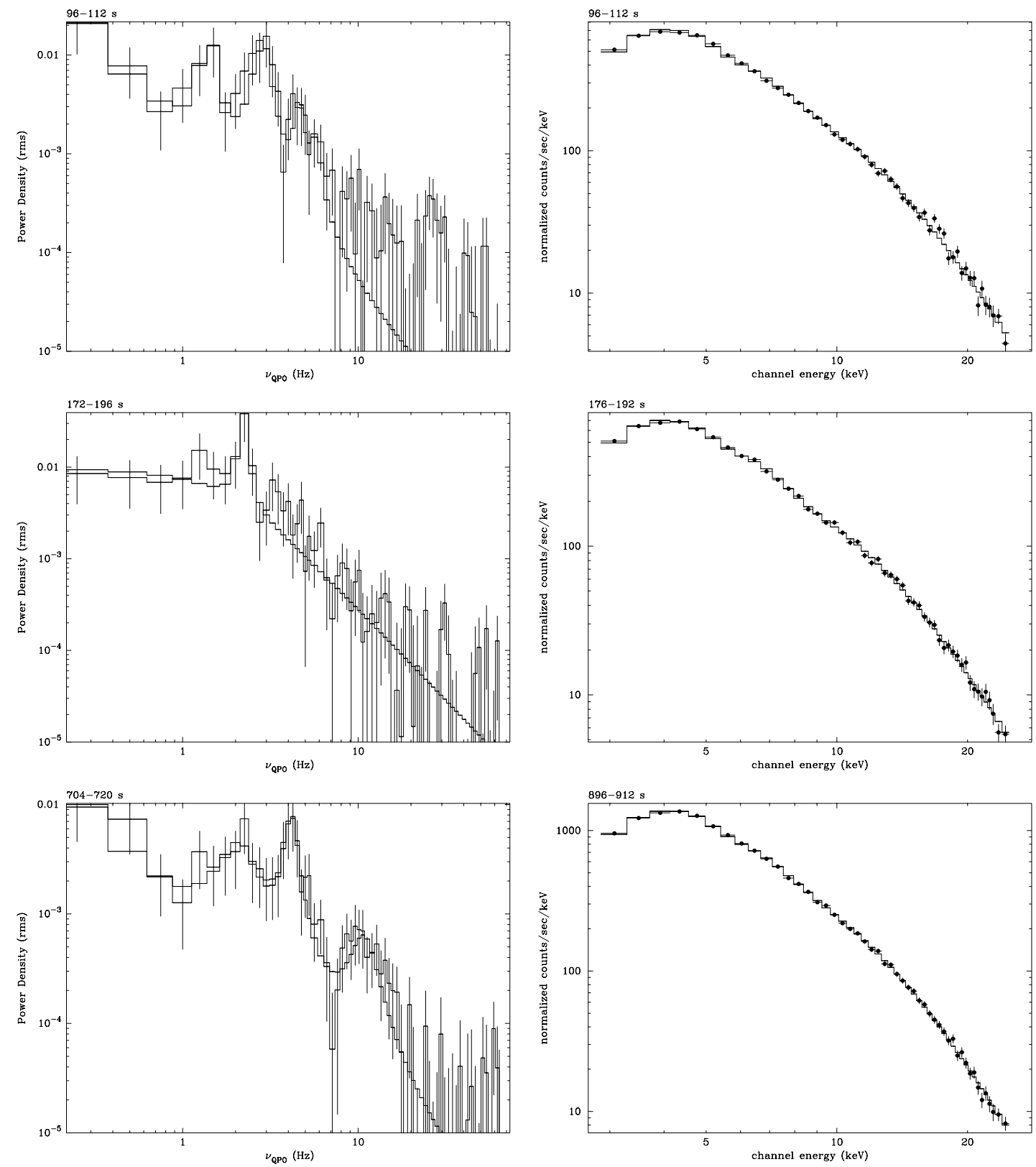

Fig. 3. Examples of power density spectra (left panels) and energy spectra (right panels) for 40702-01-02-00 (class $v$ ) corresponding to different time intervals. Start and end times for each panel are from the observation start time (Table 2).

With respect to the model adopted for the plateau observation, we needed to include a disk-blackbody (see Belloni et al. 1997b, 2000) and we had to fix $N_{\mathrm{H}}$ due to the limited statistics of the 16s spectra. Again, we added in quadrature a systematic error of $1 \%$. The fits give acceptable results, with a reduced $\chi^{2}$ usually around 1 . Therefore, we can trace the evolution (with a time step of $16 \mathrm{~s}$ ) of the spectral parameters during the state-C intervals. We can then compare the spectral and timing evolution by correlating the resulting parameters. As in the case of plateau observations we fitted selected spectra with the bmc model (see Sect. 2.2), finding consistent results for $\Gamma$.

Examples of both PDSs and energy spectra (with their best fit models) can be seen in Fig. 3. The correlation between QPO centroid frequency and power-law index for classes $\beta$ and $v$ is plotted in Fig. 5 (upper panel). The behaviour is similar to $\chi$ observations, although there is a spread due to the lower statistics available from spectra averaged over only $16 \mathrm{~s}$. At high QPO frequencies it is possible to notice a "turnoff" in the correlation for observations 15 and 16, while observation 19 (diamonds) does not show this feature. Figure 5 (lower panel) shows the same correlation from classes $\alpha$ and $v$. While $v$ data are compatible with those in the upper panel, $\alpha$ data show a correlation similar, but offset to lower photon-index values.

\section{Other sources}

In order to compare our results for GRS 1915+105 with other systems, we searched the literature for similar data from other black hole candidates. We identified four systems for which values for the QPO centroid frequency and the power-law 

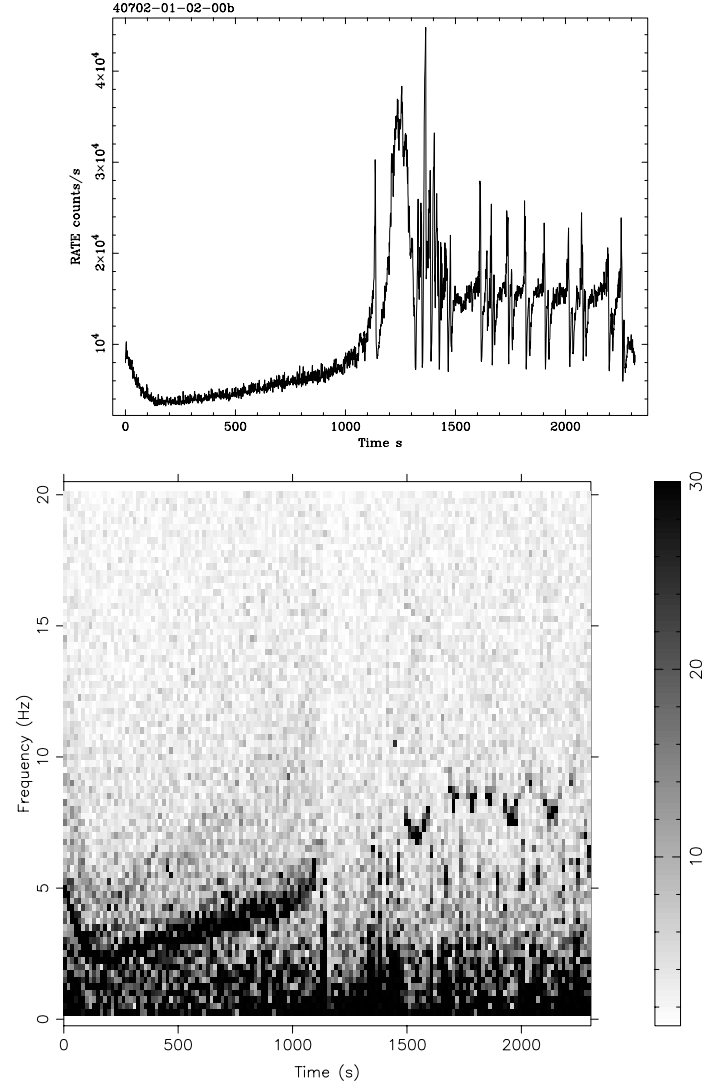

Fig. 4. Light curve and dynamical PDS for the $v$-class observation 40702-01-02-00 (second interval). It is evident that the 1-10 Hz QPO, when present, is strictly positively correlated with counts (see text). In the PDS frame, frequency is on the $y$-axis while the power scale is shown in the chromatic column on the right.

index are available, and for which there are at least a few different values. Notice that, as mentioned above, we do not consider parameters like inner disk radius, which are difficult to compare between different sources. Providing the adopted spectral models are compatible, the values of $\Gamma$ are directly comparable, and so of course are the QPO centroid frequencies.

\subsection{GRO J1655-40}

This source is a BHC with a dynamical estimate for the mass of the black hole of $\sim 6.3 M_{\odot}$ (Bailyn et al. 1995; Greene et al. 2001). The system, a bright X-ray transient which appeared for the first time in 1994 and showed a subsequent outburst in 1996/1997, showed superluminal radio jets (Hjellming \& Rupen 1995). GRO J1655-40 is therefore another of the few objects belonging to the microquasar class. This object shows quite a few different QPO peaks in a range between 0.1 and $450 \mathrm{~Hz}$, (see Remillard et al. 1999a; Strohmayer 2001; Remillard et al. 2002b). Among them, we are interested in the variable 14-22 Hz QPO (see Sobczak et al. 2000a). We consider here PCA/RXTE data from an intensive observational campaign of the source during its second outburst in 1996-1997. The timing parameters (i.e., the QPO centroid frequencies) are taken from Table 3 of Remillard et al. (1999a), and the spectral parameters (i.e., the power law photon indexes)
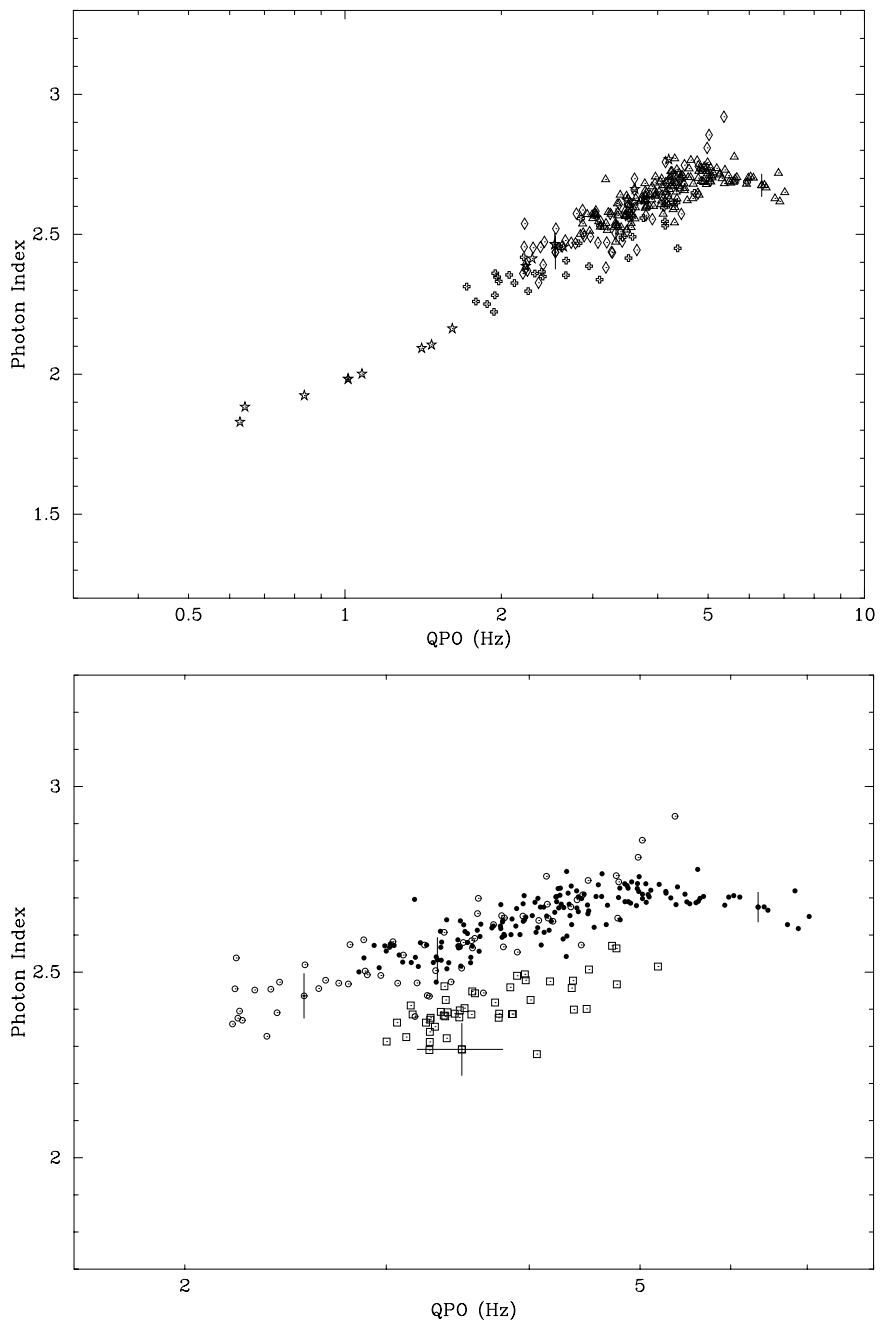

Fig. 5. Upper panel: power-law photon index vs. QPO centroid frequency for the observations of class $\beta$ and $v$ studied. Values for the plateau observations (from Fig. 2) are plotted for comparison (stars). Different symbols are used to identify different observations. [Triangles $=$ obs. 15, $16-$ Diamonds = obs. $19-$ Crosses $=$ obs. 17]. Lower panel: power-law photon index vs. QPO centroid frequency for the observations of class $\alpha$ and $v$ studied. Different symbols identify different observations. [Full circles $=$ obs. 15, $16-$ Empty circles $=$ obs. 19 - Squares = obs. 18]. In both panels error bars at $90 \%$ confidence for three data points are shown as example.

from Table 1 of Sobczak et al. (1999a). The energy spectra in the $2.5-20 \mathrm{keV}$ energy range were fitted using a model consisting of a multicolor disk-blackbody plus a power law; a detailed description of the spectral analysis can be found in Sobczak et al. (1999a). The power density spectrum for each observation was analyzed in the $2-30 \mathrm{keV}$ energy range, obtaining the QPO central frequency fitting with a Lorentzian function plus a power law for the local continuum (see Remillard et al. 1999a). The plot of power-law index versus QPO centroid frequency for GRO J1655-40 is shown in Fig. 6 (squares). There is a clear anti-correlation between these two parameters, as already shown by Sobczak et al. (1999a). 


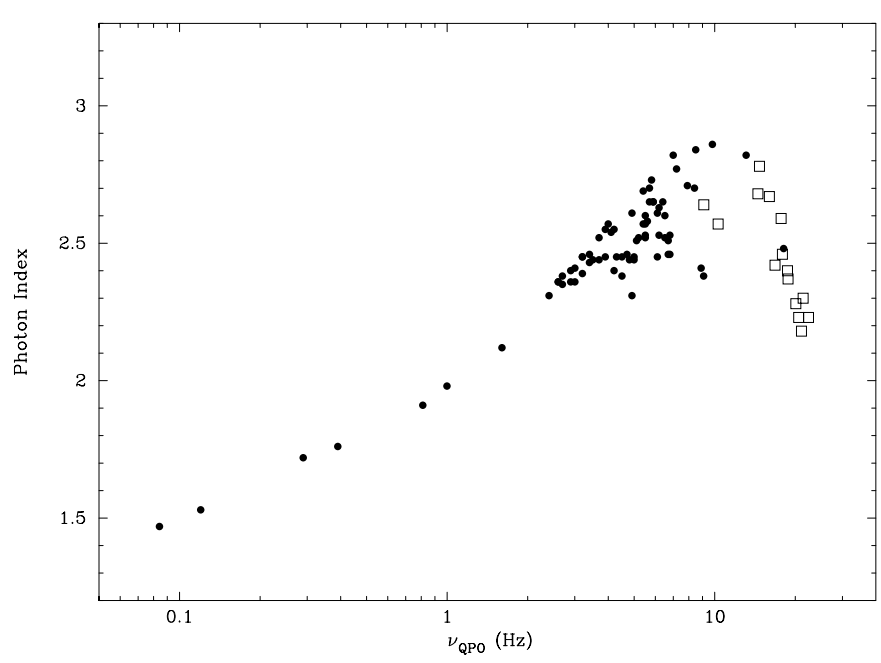

Fig. 6. Power-law photon index vs. QPO centroid frequency for $X T E$ J1550-564 (circles) and GRO J1655-40 (squares). While for the first source there is a general positive correlation between the two parameters, for the second one the correlation is negative. However, one can see that data points of GRO J1655-40 seem to extend the "turnoff" curves of XTE J1550-564 (see text).

\subsection{XTE J1550-564}

This BHC is the brightest X-ray transient observed with RXTE (the 1998-1999 flare reached $6.8 \mathrm{Crab}$ in the 2-10 keV band). In the PDS, it shows both low-frequency $(0.8-18 \mathrm{~Hz})$ and highfrequency (180-280 Hz) QPOs (Cui et al. 1999; Remillard et al. 1999b; Homan et al. 2001; Remillard et al. 2002a,b). We consider here the low-frequency one (see Remillard et al. 2002a). The observations presented here are the whole set made with PCA/RXTE before 1999 March 22 (Gain Epoch 3). The power-law photon indexes obtained from the spectral analysis of these observations are taken from Table 1 of Sobczak et al. (1999b), while the QPO centroid frequencies from the PDS analysis are listed in Table 1 of Sobczak et al. (2000a). The spectral model used by Sobczak et al. (1999b) to fit the 2.5-20 keV energy spectra consists again of the superposition of a multicolor disk-blackbody and a power law, with corrections for interstellar absorption and a Gaussian emission line at $\sim 6-7 \mathrm{keV}$. The centroid frequency of the QPO was found fitting the peak with a Lorentzian function and the continuum with a power law.

Data obtained from the literature for XTE J1550-564 are plotted in Fig. 6 (circles). They show a positive correlation between power law photon index and QPO centroid frequency very similar to the behaviour of GRS 1915+105 (see Figs. 2 and 5). However, while the correlation is very good at low frequency, at higher QPO values there is a larger spread. Surprisingly, the points from GRO J1655-40, although showing a negative correlation, are placed on the natural continuation of the upper points for XTE J1550-564. As one can see from Cui et al. (1999), Remillard et al. (2002b) and Homan et al. (2001), the low-frequency QPO in XTE J1550-564 does not maintain the same properties throughout the whole outburst. It is therefore natural to examine its behaviour in separate intervals of the light curve.

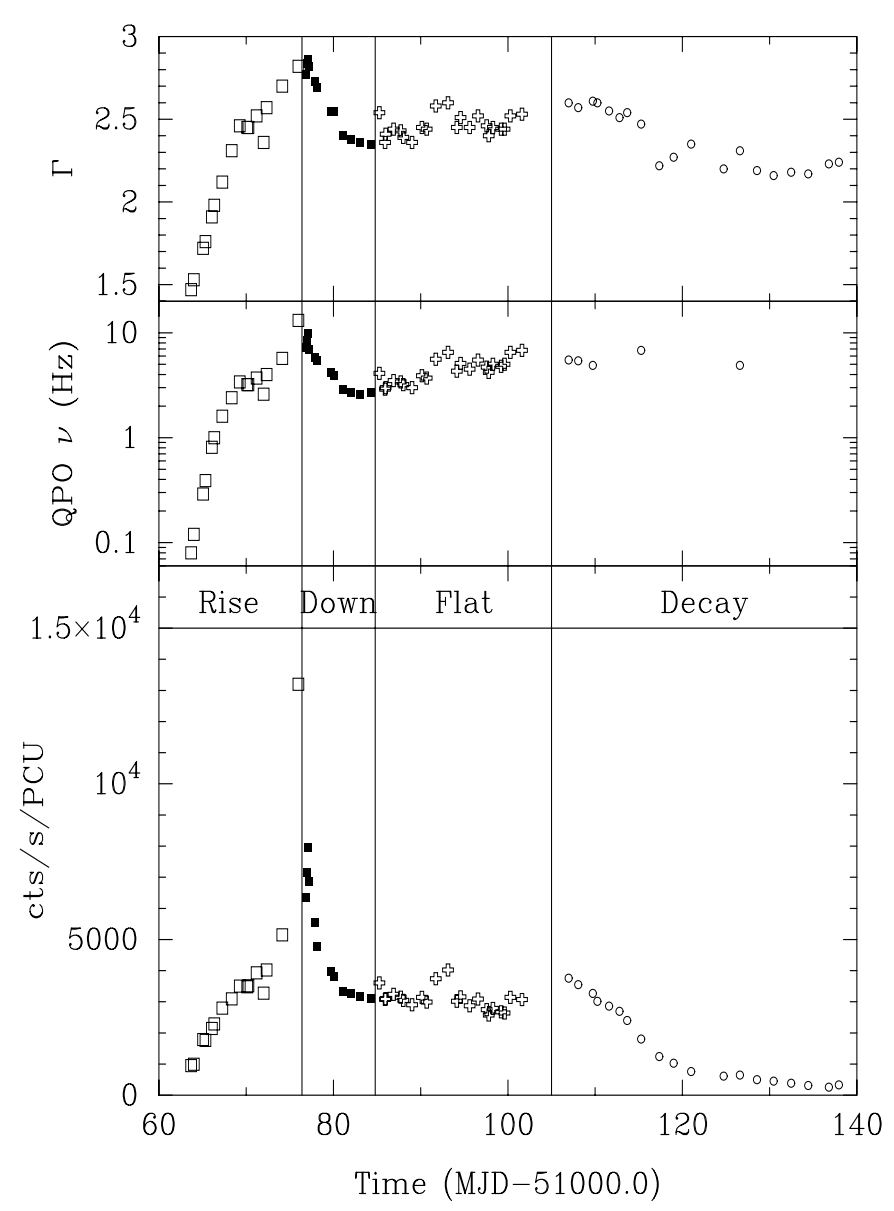

Fig. 7. Bottom panel: light curve for XTE J1550-564 as derived from Table 1 of Sobczak et al. (2000b). Observations span from 7th September 1998 to 20th November 1998. Different symbols correspond to the different intervals marked in the panel (see also Fig. 8). Middle panel: corresponding QPO frequencies (from Sobczak et al. 2000a). Top panel: corresponding power-law slopes (from Sobczak et al. 1999b).

We subdivide the light curve in four separate sections (see Fig. 7): a "rise" interval from the first observation up to the peak flux, a "down" interval from the peak following the smooth decrease in flux until the first small flare, a "flat" interval where the flux remains rather stable, with small flares, and a "decay" interval where the flux decays at the end of the first part of the outburst. After the last observation examined here, no QPO was detected for a long time, until the flux rose again to the second part of the outburst (see Homan et al. 2001).

In Fig. 8 the behaviour of XTE J1550-564 in the QPO- $\Gamma$ plane is shown with different symbols for the four different intervals. One can see that the first two intervals trace a very smooth curve in Fig. 8, the second interval tracing back the path of the first. The scattered points correspond to the remaining two intervals, and it is interesting to note that all these points are located below the others. What is different between the first two and the second two classes of observations that have a different behaviour in this plot? From Sobczak et al. (2000b) one can see that a clear dividing line between the two groups is the ratio of the power-law flux over the total flux, estimated from the observed PCA spectra. The first observations have a lower 


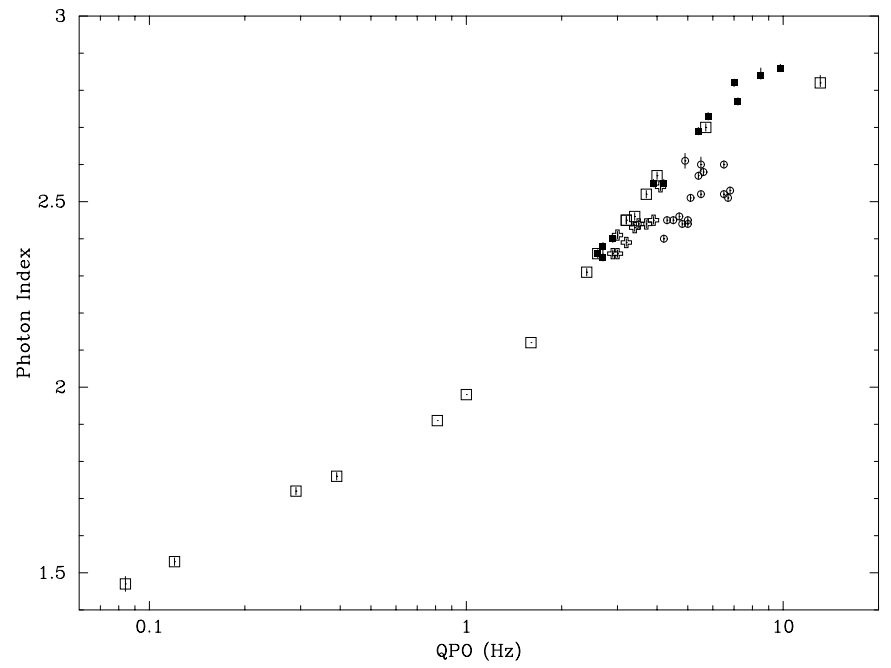

Fig. 8. Plot of power law photon index versus QPO centroid frequency for XTE J1550-564. Data points are labeled with different marks according to the selection explained in text (same symbols as in Fig. 7).

value of the ratio. Also notice that the points that contribute to the scatter in Fig. 8 are the ones from the "decay" interval, which contains QPOs classified as A/B in Homan et al. (2001), while the QPO observed in the previous intervals is classified as $\mathrm{C}$ (notice that these QPO classes have nothing to do with the $\mathrm{A} / \mathrm{B} / \mathrm{C}$ classes defined in Belloni et al. 2000).

\subsection{XTE J1748-288}

This BHC shows superluminal radio jets and belongs to the class of galactic microquasars, for the same reasons as GRS 1915+105 and GRO J1655-40 (Smith et al. 1998; Hjellming et al. 1998; Revnivtsev et al. 2000; Kotani et al. 2000; Miller et al. 2001). During the 1998 outburst the source was observed to display spectral and timing properties typical for BHCs systems (Revnivtsev et al. 2000). A QPO feature was found in the PDS at $0.5 \mathrm{~Hz}$ and $20-30 \mathrm{~Hz}$. Here we consider spectral and timing parameters given in Tables 2 and 3 of Revnivtsev et al. (2000) who reported an analysis of the PCA/RXTE observations during the 1998 outburst. They fitted the energy spectra between 3 and $25 \mathrm{keV}$ using the standard model for BHCs consisting of a multicolor disk-blackbody plus a power law, with low energy interstellar absorption and a Gaussian emission line at $\sim 6.5 \mathrm{keV}$. For the seven observations considered here, Revnivtsev et al. (2000) fitted the PDS using the sum of a flat-topped band limited noise component, a power law component, and a Lorentzian function to describe the QPOs. The resulting points in the QPO- $\Gamma$ plot are shown as empty diamonds in Fig. 9. There is a clear positive correlation between $\Gamma$ and QPO, but it is not identical to the same correlations of the three sources previously discussed. Notice that the QPO frequencies are the highest considered here.

\section{4. $4 U 1630-47$}

This X-ray transient is considered a BHC for which the optical thin radio emission, observed during the 1998 outburst,

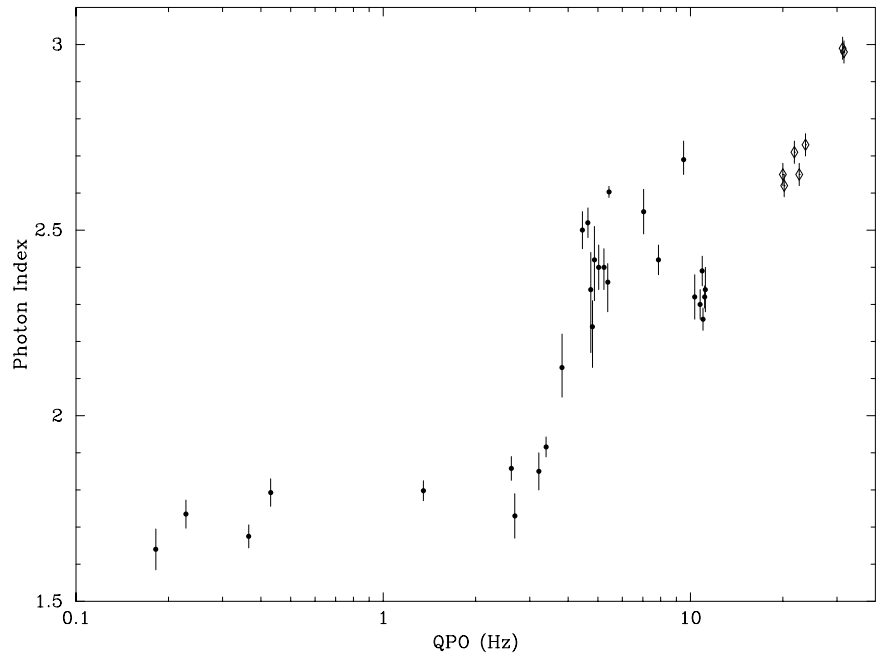

Fig. 9. Plot of power law photon index versus QPO centroid frequency for XTE J1748-288 (diamonds) and 4U 1630-47 (filled circles).

suggests the presence of radio lobes (Hjellming et al. 1999). We obtained timing (QPO centroid frequency) and spectral (power-law index) parameters of the observations made during the decay of the 1998 outburst from Tables 4 and 5 of Tomsick \& Kaaret (2000). The timing analysis was made in the 2-21 keV energy band fitting the PDS with a flat-top component for the continuum and a Lorentzian function for the QPO feature. The centroid frequency was between 0.2 and $3.4 \mathrm{~Hz}$. The energy spectra were fitted in the $2.5-20 \mathrm{keV}$ range using a power law, a multicolor disk-blackbody soft component, a Gaussian emission line at $\sim 7 \mathrm{keV}$, the interstellar absorption and a broad iron absorption edge. For the peak of the outburst, we used the results of Trudolyubov et al. (2001). Here timing analysis was performed from data in the $2-13 \mathrm{keV}$ energy range and the PDS were fitted with Lorentzian models plus a low frequency power-law. The PDS were rather complex: as centroid frequency we used the peak at the lowest frequency in the $1-20 \mathrm{~Hz}$ range. The 3-20 keV PCA energy spectra were fitted with a disk-blackbody plus a power law, although the presence of an excess around 6-8 keV was recognized.

The points corresponding to this source are shown in Fig. 9. It is possible to recognize a behaviour similar to those shown by GRS $1915+105$ and XTE J1550-564, although in this case the correlation is not as smooth. The points corresponding to the end phase of the outburst have a rather constant $\Gamma$ around 1.7-1.8, while the QPO frequency decreases from $3 \mathrm{~Hz}$ down to $0.2 \mathrm{~Hz}$ (data from Tomsick \& Kaaret 2000). Notice that the points corresponding to the lowest frequencies $(0.2$ to $0.5 \mathrm{~Hz})$ do match rather well the correlation followed by XTE J1550564. At higher QPO frequencies there is a correlation between the two quantities, with a rather large scatter and a possible indication of a turn-off.

\section{Summary and discussion}

Our results can be summarized as follows. For the $\chi$ class of GRS $1915+105$, when the energy spectrum is rather hard and consists exclusively of the power-law component and the 


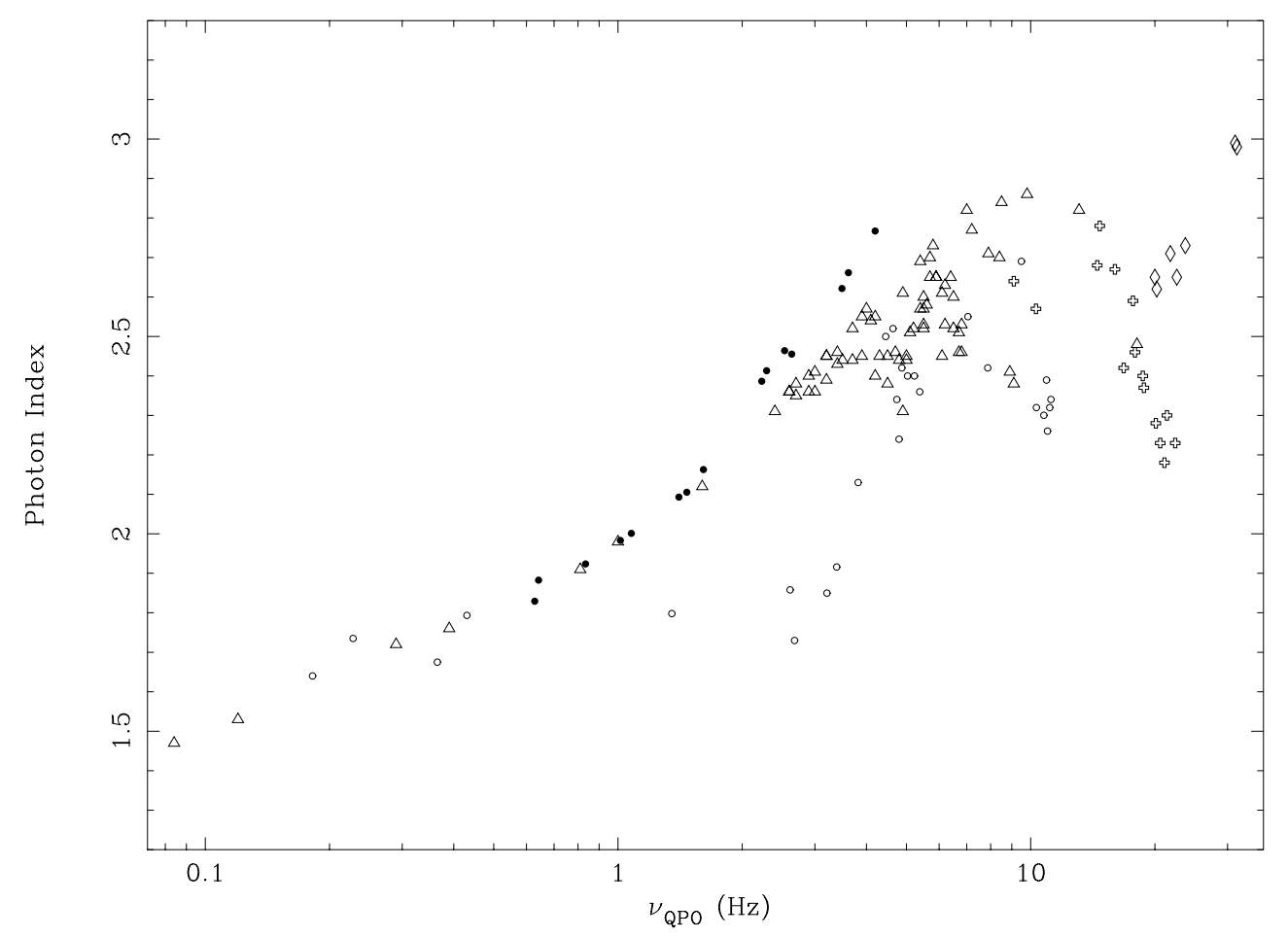

Fig. 10. Plot of power law photon index versus QPO centroid frequency. Plateau results from GRS $1915+105$ are plotted in full circles; empty crosses are data from GRO J1655-40; empty circles are data from 4U 1630-47; diamonds are data from XTE J1748-288; empty triangles are data from XTE J1550-564.

power density spectrum shows a clear $1-10 \mathrm{~Hz}$ QPO, there is a strong positive correlation between QPO centroid frequency and photon index of the power law. At the highest values of the QPO centroid frequencies, this correlation flattens and appears to start reversing. Published data from other sources indicate that this general correlation exists for a number of sources, and indeed in some cases there is a turnoff in the relation for high QPO frequencies. The correlation is not exactly the same for all sources and in the case of XTE J1550-564 there is evidence for different turnoffs during different intervals of the source outburst.

The turnoff in the correlation is difficult to understand. If the QPO frequency is associated to a specific radius in the accretion disk, only the rising part of the correlation can be explained with a simple Comptonization model. If the QPO-radius relation also shows a reversal (see for instance Rodriguez et al. 2002 and Varniére et al. 2002), then a turnoff like that presented here can be produced.

The source for which we have more data, and the largest range in QPO frequencies is XTE J1550-564. From Fig. 8 one can see that not only the points from the first two sections of the outburst (see Fig. 7) follow a very narrow correlation, but also that all subsequent points lie below this correlation. This suggests that the correlation followed in the first part, when the overall PDS was in the form of a flat-top noise component plus a QPO (Cui et al. 1999; Remillard et al. 2002b), is perturbed in a very specific way when the source changes state and a powerful disk component appears in the energy spectrum (Sobczak et al. 1999b, 2000a,b). There is increasing evidence that the power-law component observed in the energy spectra of BHCs in the Low/Hard state is physically different from that seen in the Very High State (see e.g. Zdziarski et al. 2002). It is therefore possible that the turnoff is due to a gradual transition between these two regimes.

It is interesting to discuss the position of the turnoff in the QPO- $\Gamma$ relation. Figure 10, where all the sources are shown together, suggests that: (a) XTE J1550-564 and GRO J1655-40 have a similar turnoff frequency around $10 \mathrm{~Hz}$; (b) XTE J1748-288 has a higher turnoff frequency, if any, larger than $30 \mathrm{~Hz}$; (c) GRS 1915+105 (see Fig. 5) has a lower turnoff frequency at about $5 \mathrm{~Hz}$. It is tempting to compare these values with the relative values of the estimated dynamical masses for the compact objects in the systems. Excluding XTE J1748-288, for which no dynamical mass estimate is available, we see that GRS $1915+105$ has a mass roughly twice and a turnoff frequency half that of GRO J1655-40, as one could expect with a simple mass scaling. However, the recent mass estimate for XTE J1550-564 is around $10 M_{\odot}$ (Orosz et al. 2002), from which we would expect a turnoff frequency lower by a factor of $\sim 2$ with respect to that of GRO J1655-40, which is evidently not observed.

In conclusion, we have found that in GRS $1915+105$ and a number of other BHCs, the centroid frequency of the lowfrequency QPO and the photon index of the power-law component in the energy spectra follow a very similar kind of correlation. In particular, for some sources there is evidence for the presence of a turnoff in this correlation. If the QPO frequency is associated to a specific radius of the accretion disk, this provides an important clue to the possible origin of the power-law component. More data are needed in order to 
understand the physical nature of the turnoff, but these results stress the importance of such simple spectral/timing correlations for black hole candidates.

Acknowledgements. TB acknowledges the hospitality of MIT and thanks the Cariplo Foundation for financial support.

\section{References}

Bailyn, C. D., Orosz, J. A., McClintock, J. E., \& Remillard, R. A. 1995, Nature, 378, 157

Belloni, T., \& Hasinger, G. 1990, A\&A, 230, 103

Belloni, T., Méndez, M., King, A. R., van der Klis, M., \& van Paradijs, J. 1997a, ApJ, 479, L145

Belloni, T., Méndez, M., King, A. R., van der Klis, M., \& van Paradijs, J. 1997b, ApJ, 488, L109

Belloni, T., Abstracts of the 19th Texas Symposium on Relativistic Astrophysics and Cosmology, held in Paris, France, Dec. 14-18, 1998, ed. J. Paul, T. Montmerle, \& E. Aubourg (CEA Saclay), E325

Belloni, T., Klein-Wolt, M., Méndez, M., van der Klis, M., \& van Paradijs, J. 2000, A\&A, 355, 271

Belloni, T., Sánchez-Fernández, C., \& Méndez, M. 2001, A\&A, 372, 551

Belloni, T., Psaltis, D., \& van der Klis, M. 2002, ApJ, 572, 392

Borozdin, K., Revnivtsev, M., Trudolyubov, S., Shrader, C., \& Titarchuk, L. 1999, ApJ, 517, 367

Castro-Tirado, A. J., Brandt, S., \& Lund, S. 1992, IAU Circ., 5590

Chen, X., Swank, J. H., \& Taam, R. E. 1997, ApJ, 477, L41

Cui, W., Zhang, S. N., Chen, W., \& Morgan, E. H. 1999, ApJ, 512, L43

Di Matteo, T., \& Psaltis, D. 1999, ApJ, 526, L101

Fender, R. P., Garrington, S. T., McKay, D. J., et al. 1999, MNRAS, 304, 865

Greene, J., Bailyn, C. D., \& Orosz, J. A. 2001, ApJ, 554, 1290

Greiner, J., Cuby, J. G., \& McCaughrean, M. J. 2001, Nature, 414, 522

Hjellming, R. M., \& Rupen, M. P. 1995, Nature, 375, 464

Hjellming, R. M., Rupen, M. P., Mioduwszewski, A. J., et al. 1998, BAAS, 30, 1405

Hjellming, R. M., Rupen, M. P., Mioduwszewski, A. J., Kuulkers, E., \& McCollough, M. 1999, ApJ, 514, 383

Homan, J., Wijnands, R., van der Klis, M., Belloni, T., \& van Paradijs, J. 2001, ApJS, 132, 377

Klein-Wolt, M., Fender, R. P., Pooley, G. G., et al. 2002, MNRAS, 331,745

Kotani, T., Kawai, N., Nagase, F., et al. 2000, ApJ, 543, L133

Markwardt, C. B., Swank, J. H., \& Taam, R. E. 1999, ApJ, 513, L37

Merloni, A., Fabian, A. C., \& Ross, R. R. 2000, MNRAS, 313, 193

Migliari, S., Vignarca, F., \& Belloni, T. 2001, ApSSS, 276, 183

Miller, M. C., Lamb, F. K., \& Psaltis, D. 1998, ApJ, 508, 791

Miller, J. M., Wijnands, R., Homan, J., et al. 2001, ApJ, 563, 928

Mirabel, I. F., \& Rodríguez, L. F. 1994, Nature, 371, 46

Mirabel, I. F., Duc, P. A., Rodríguez, L. F., et al. 1994, A\&A, 330, L9
Mitsuda, K., Inoue, H., Koyama, K., et al. 1984, PASJ, 36, 741

Morgan, E., Remillard, R. A., \& Greiner, J. 1997, ApJ, 482, 993

Muno, M. P., Morgan, E. H., \& Remillard, R. A. 1999, ApJ, 527, 321

Nobili, L., Turolla, R., Zampieri, L., \& Belloni, T. 2000, ApJ, 538, L137

Nowak, M. A., \& Wagoner, R. V. 1991, ApJ, 378, 656

Orosz, J. A., Groot, P. J., van der Klis, M., et al. 2002, ApJ, 568, 845

Psaltis, D., \& Norman, C. 2002, ApJ, in press [astro-ph/0001391]

Reig, P., Belloni, T., van der Klis, M., et al. 2000, ApJ, 541, 883

Remillard, R. A., Morgan, E. H., McClintock, J. E., Bailyn, C. D, \& Orosz, J. A. 1999a, ApJ, 522, 397

Remillard, R. A., McClintock, J. E., Sobczak, G. J., et al. 1999b, ApJ, 517, L127

Remillard, R. A., Muno, M. P., McClintock, J. E., \& Orosz, J. A. 2002a, ApJ, submitted [astro-ph/0202305]

Remillard, R. A., Sobczak, G. J., Muno, M. P., \& McClintock, J. E. 2002b, ApJ, 564, 962

Revnivtsev, M. G., Borozdin, K. N., Priedhorsky, W. C., \& Vikhlinin, A. 2000, MNRAS, 312, 151

Rodriguez, J., Varniére, P., Tagger, M., \& Durouchoux, Ph. 2002, A\&A, 387, 487

Smith, D. A., Levine, A., \& Wood, A. 1998, IAUC, 6932

Sobczak, G. J., McClintock, J. E., Remillard, R. A., Bailyn, C. D., \& Orosz, J. A. 1999a, ApJ, 520, 776

Sobczak, G. J., McClintock, J. E., Remillard, R. A., et al. 1999b, ApJ, 517, L121

Sobczak, G. J., McClintock, J. E., Remillard, R. A., et al. 2000a, ApJ, 531,537

Sobczak, G. J., McClintock, J. E., Remillard, R. A., et al. 2000b, ApJ, 544, 993

Stella, L., \& Vietri, M. 1998, ApJ, 492, L59

Stella, L., Vietri, M., \& Morsink, S. M. 1999, ApJ, 524, L63

Strohmayer, T. E. 2001a, ApJ, 554, L169

Strohmayer, T. E. 2001b, ApJ, 552, L49

Titarchuk, L., Lapidus, I., \& Muslimov, A. 1998, ApJ, 499, 315

Tomsick, J. A., \& Kaaret, P. 2000, ApJ, 537, 448

Trudolyubov, S. P., Churazov, E. M., \& Gilfanov, M. R. 1999, Astr. Lett., 25, 718

Trudolyubov, S. P., Borozdin, K. N., \& Priedhorsky, W. C. 2001, MNRAS, 322, 309

van der Klis, M. 1995, in X-ray binaries, ed. W. H. G. Lewin, J. van Paradijs, \& E. P. J. van den Heuvel (Cambridge Univ. Press, Cambridge), p. 252

Varniére, P., Rodriguez, J., \& Tagger, M. 2002, A\&A, 387, 497

Tanaka, Y., \& Lewin, W. H. G. 1995, in X-ray binaries, ed. W. H. G. Lewin, J. van Paradijs, E. P. J. van den Heuvel (Cambridge Univ. Press, Cambridge), 126

Zdziarski, A. A., Poutanen, J., Paciesas, W. S., \& Wen, L. 2002, ApJ, 578,357

Zhang, W. 1995, RXTE/PCA Internal memo, 5-23-95

Zhang, W., Jahoda, K., Swank, J. H., Morgan, E. H., \& Giles, A. B. 1995, ApJ, 449, 930 